\title{
Thermography based in-process monitoring of Fused Filament Fabrication of polymeric parts
}

\author{
E. Ferraris, J. Zhang, B. Van Hooreweder \\ Department of Mechanical Engineering, KU Leuven, Belgium \\ Submitted by Han Haitjema <han.haitjema@kuleuven.be>
}

Temperature management is crucial in Fused Filament Fabrication (FFF). This paper presents an infrared based set-up able to record spatial and temporal variations during printing of an FFF part, with a field of view of $22 \times 15$ (mm) at spatial resolution of $30.2 \mu \mathrm{m}$. The system is experimentally verified and the captured temperature profiles are compared to numerical data, obtained via an own-developed FFF numerical model. This work is a first step towards a combined experimental-numerical approach for in-process monitoring of FFF, with implications for filament developers, machine builders and (end-) users, ultimately demanding high quality (certified) parts.

Additive manufacturing, fused deposition, in-process measurement

\section{Introduction}

Fused Filament Fabrication (FFF) (also known as Fused Deposition Modeling, FDM $^{\mathrm{TM}}$ ) is a widely used additive manufacturing (AM) technology to produce polymeric parts (Galantucci et al. [1]). It involves extrusion of a thermoplastic filament through a heated nozzle and deposition of the molten extrudate onto a build platform along a given tool path. The part forms due to coalescence of the deposited tracks (or filament segments), and it is constructed layer by layer.

According to Bellehumeur et al. [2], coalescence of contiguous filament segments develops over a process of contact, wetting and neck growth, which is driven by either sintering or thermal diffusion of adjacent particles at temperatures above the material glass-transition temperature $\left(T_{\mathrm{g}}\right)$. In addition, neck growth is a function of time, surface energy and material viscosity (both temperature-dependent), and of the track diameter; this latter being of small size (typically $<300 \mu \mathrm{m}$ ) and therefore inducing rapid temperature variation (high cooling/heating rate) (Volz, [3]). Hence, FFF is inherently a thermally driven process.

Geometrical accuracy, surface finishing, mechanical strength, macro-, micro-porosity, etc. of a printed part are then the result of the spatial and temporal distribution of temperature during printing (thermal history), which is implicitly affected by the input parameters (filament material, nozzle temperature, print speed, filling strategy, deposition sequence, nozzle diameter, layer thickness, etc.). Faes et al. [4] correlated the mechanical performance of ABS components with the fabrication strategy selected and number of parts to print in a build via the inter-layer cooling time (i.e. the time interval for material to cool between printing of two consecutive layers in a given part). Similarly, print speed, filling sequence and part dimension affect the temperature field during printing, given their influence on the intra- and inter-layer time (i.e. the time interval between printing adjacent tracks in the same layer and between consecutive layers, respectively). Sun et al. [5] concluded that fabrication strategy, environment temperature and variations in convection determine the overall quality of the bond strength due to their effect on cooling rate. In general, a filament segment should quickly solidify to avoid excessive deformation due to gravity; conversely, it should remain hot sufficiently long to ensure adequate bonding with the neighbouring filament segment(s) (Costa et al. [6]).

In the literature, several works address the study of temperature evolution in FFF. Rodriguez et al. [7] developed a 2D transient heat transfer analysis to study solidification of a filament in a vertical stack. They concluded that lower cooling rates promote stronger bonding. Bellini and Güceri [8] used a finite element method (FEM) to model extrusion, deposition and cooling stages of FFF at various inputs, while Costa et al. [6] proposed a coupled analytical and computational approach to predict bond quality between adjacent filaments in an FFF part. Experimental monitoring of temperature has also been revealed particularly challenging in FFF, due to the characteristic small dimensions (100-300 $\mu \mathrm{m})$ and the additive nature of the process. Kousiatza and Karalekas [9] utilised thermocouples and fibre Bragg grating sensors, but experimental observations are limited to local measurements. In addition, the sensors would need to be very small (few tens of micrometers) in order not to interfere with the targeted temperature. Due to the noncontact nature of the sensing principle, infrared thermography is a promising alternative instead. Some recent attempts of its applications can be found in Wolszczak et al. [10] and Malekipour et al. [11], but they lack verification and critical insights into the achievable accuracy.

To conclude, research on the development of FFF inprocess monitoring approach is still in an early stage. As a first attempt towards the development of a combined experimental-numerical approach, this work presents an infrared based set-up able to record three-dimensional (2D space and time) temperature variations during printing of an FFF part, along with its experimental validation and assessment against numerical data. 


\section{Materials and methods}

An Optris PI640 infrared camera was used in this research. Technical data are: wavelength range 7.5-13 $\mu \mathrm{m}$; sampling frequency $32 \mathrm{~Hz}$; optical resolution $640 \times 480$ pixels; spatial resolution $30.2 \mu \mathrm{m} /$ pixel and field of view (FOV) $21.7 \times 14.9 \mathrm{~mm}$ at a working distance of $85 \mathrm{~mm}$. Given the high spatial resolution, up to 9 measurements could be taken within a single track of $0.3 \mathrm{~mm}$ diameter. The camera was mounted was mounted on a tri-pod facing the build platform of a commercial Prusa MK3 (Fig.1-i). For an object aligned with the cartesian system of the machine, the camera then captures temporal temperature variations in the object front $x-z$ plane. Hence, temperature changes at a given location $\left(x_{i} y_{j} z_{k}\right)$ due to primary re-heating (i.e. due to deposition of

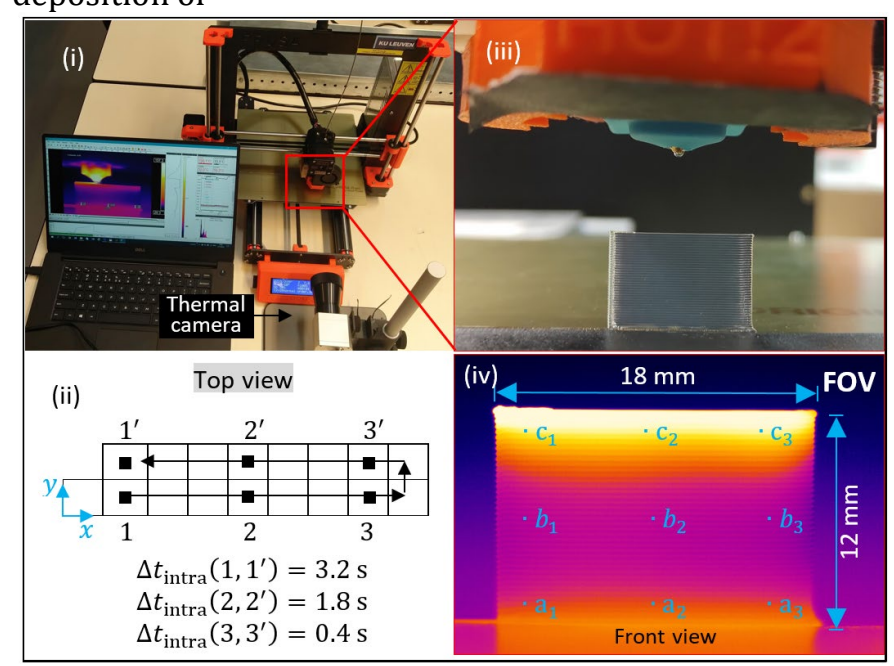

Fig. 1. i) Picture of the thermal set-up for in-process monitor of the FFF process; ii) top view of the sample geometry, deposition sequence and intra-layer time

( $\Delta t_{\text {intra }}$ ) for elements located on given $x-y$ planes; iii) picture of a realised sample and iv) thermogram of the printed part. Given $x-z$ locations are highlighted.

the top neighbour $\left(x_{i} y_{j} z_{k+1}\right)$ or of the back neighbour $\left.\left(x_{i} y_{j+1} z_{k}\right)\right)$ and due to secondary re-heating (i.e. due to deposition of the neighbour of the neighbour $\left.\left(x_{i} y_{j+1} z_{k+1}\right)\right)$ are also visible. The nozzle mantel was shielded and the build platform was sprayed with "non-reflective" paint (LabIR ${ }^{\circledR}$ Paint, HERPLT-MWIR-BK-11, reflectivity $R \leq 0.08$ ) in order to filter the thermal signal of the object.

In-process monitoring was conducted on a double wall (2 tracks) sample geometry, $18 \times 0.8 \times 12 \mathrm{~mm}^{3}$. Once produced, it covers the FOV almost completely. FFF settings are as depicted in Table 1; the layer nozzle path is given in Fig. 1ii. The inter-layer time ( $\left.\Delta t_{\text {inter }}\right)$ is $3.6 \mathrm{~s}$, while the intra-layer time $\left(\Delta t_{\text {intra }}\right)$ depends on the particular location of the considered filament elements in the $y-z$ plane. Fig. 1-iii shows an image of a realised sample along with its thermogram (Fig.1-iv).
A material track was deposited onto the build platform heated at $60^{\circ} \mathrm{C}$; and the material emissivity, $\varepsilon$, was calibrated by minimising the absolute difference of the track's steady state temperatures measured by the thermal camera $T(\varepsilon)$ and a thermocouple $\left(60.4^{\circ} \mathrm{C}\right)$.

$$
\min _{\varepsilon \in[0,1]}\left|T(\varepsilon)-60.4^{\circ} \mathrm{C}\right|,
$$

An effective emissivity $\varepsilon=0.78$ was obtained. In this context, it must be noted that the emissivity increases with the temperature but decreases with the view angle $\alpha$ of the thermal camera. Specifically, $\alpha$ is here defined as the angle between the direction of thermal radiation received by the camera and the normal to the surface $(\boldsymbol{n})$ on a given point (see Fig. 4). Accordingly, due to the characteristic wave-like profile of an FFF part, the data measured in the proximity of the concave regions are underestimated because of the surface local orientation $\left(\alpha \sim 90^{\circ}\right)$. To compensate for this, the track temperature is assumed constant across the crosssection and it is computed by averaging the 6 highest values. Recorded temperature profiles were compared to numerical data. The model adopted in this research was previously developed by Zhang et al. in [12]. It is based on a boundary adjust finite difference method (FDM) and it is capable to solve three-dimensional temperature variations in FFF given the nozzle diameter, nozzle temperature, air temperature, build platform temperature, printing speed, layer thickness, object dimensions $(L \times W \times H)$, at fixed raster angle $0^{\circ}$ and filling ratio $100 \%$. A FFF part is assumed to be a sequence of ideal material blocks, and the governing equation in the time $(t)$-dependent geometry domain $\Omega$ reduces to:

\section{Table 1}

Parameter setting for sample printing.

\begin{tabular}{ll}
\hline Parameters & Values \\
\hline Model dimension $L \times W \times H$ & $18 \times 0.8 \times 12 \mathrm{~mm}^{3}$ \\
Layer thickness $\Delta h$ & $0.3 \mathrm{~mm}$ \\
Printing speed $v$ & $10 \mathrm{~m} / \mathrm{s}$ \\
Nozzle temperature $T_{\mathrm{n}}$ (nominal) & $215^{\circ} \mathrm{C}$ \\
Platform temperature $T_{\mathrm{p}}$ (nominal) & $60{ }^{\circ} \mathrm{C}$ \\
Air temperature $T_{\mathrm{a}}$ & $21.2^{\circ} \mathrm{C}$ \\
Fan speed & $O N-\max$ speed \\
Nozzle diameter (E3D V6 nozzle & $0.4 \mathrm{~mm}$ \\
type) & \\
PLA filament diameter (PRUSA & $1.75 \mathrm{~mm}$ \\
supplier) & \\
\hline
\end{tabular}

$$
\rho c_{\mathrm{p}} \frac{\partial T}{\partial t}=\lambda\left(\frac{\partial^{2} T}{\partial x^{2}}+\frac{\partial^{2} T}{\partial y^{2}}+\frac{\partial^{2} T}{\partial z^{2}}\right), x \in \Omega(t)
$$

by assuming homogeneous thermal diffusion and weak dependence of the conduction coefficient $\lambda$ on temperature $T$. Material density $\rho$ and specific heat capacity $c_{\mathrm{p}}$ are temperature-dependent physical properties, and the boundary conditions are of the first type. For additional information, see [12]. For the purpose of this research, the process parameters were set as given in Table 1 . The nominal temperature values were adjusted to measured data (obtained with thermocouples); and the air 
temperature was set as a function of the vertical distance from the build platform. The polylactic acid (PLA) material properties are as given in [12].

\section{Experimental Results}

The experimental and numerical temperature profiles were compared to each other in the space and in the time domain.

Fig. 2 shows the temperature evolution in the time for 9 selected locations, distributed in the $x-z$ plane, and respectively located at the bottom (a), centre (b) and top (c) of the object and at the left (1), central (2) and right (3) side.

Although discrepancies in the absolute values are present, a good match between the numerical and experimental pattern is observed. Local temperature decreases rapidly after deposition; successively, a number of peaks due to primary and secondary re-heating are experimentally recorded and numerically predicted at matched intervals. Less than $2 \mathrm{~min}$ is typically sufficient to reach thermal equilibrium with the environment and build platform.

The temperature peaks recorded by the thermal camera at time of deposition and for a given location (marked with a red arrow), are highly underestimated as compared with the numerical predictions and expectations (i.e. about the nozzle temperature). Those differences decrease with the number of deposited layers. As a general trend, the experimental temperature profile rise with the number of the deposited layers.

The sequences of temperature peaks due to re-heating are in agreement with the intra- and inter-layer times for the given location. For example, looking at the temperature profile of Fig. $2-\mathrm{a}_{2},\left(\Delta t_{\text {intra }}=1.8 \mathrm{~s}\right.$, i.e. an half of $\left.\Delta t_{\text {inter }}=3.6 \mathrm{~s}\right)$, a first rise in temperature is registered after $1.8 \mathrm{~s}$ (marked with a yellow arrow) due to deposition of the back neighbour, and a second peak of temperature is indicated at $3.6 \mathrm{sec}$ (marked with a blue arrow) due to deposition of the top neighbour. Increasingly weaker peaks take place afterwards due to secondary re-heating (i.e. deposition of the neighbour of the neighbour). Typically, the penetration depth due to secondary re-heating is limited to 1-2 layers (or tracks) depending on the particular conditions of material, track diameter, layer thickness, etc. Contrary to the left side, (location $\mathrm{a}_{3}, \mathrm{~b}_{3}$, and $\mathrm{c}_{3}$ ) the first re-heating takes place quickly after deposition at the right side, due to the short intra-layer time $(0.4 \mathrm{~s})$; the material is still above 60 ${ }^{\circ} \mathrm{C}$ and remains "hot" for a relatively long period. This type of temperature profile should be representative of a long bond line between adjacent tracks (see also Sect.5).

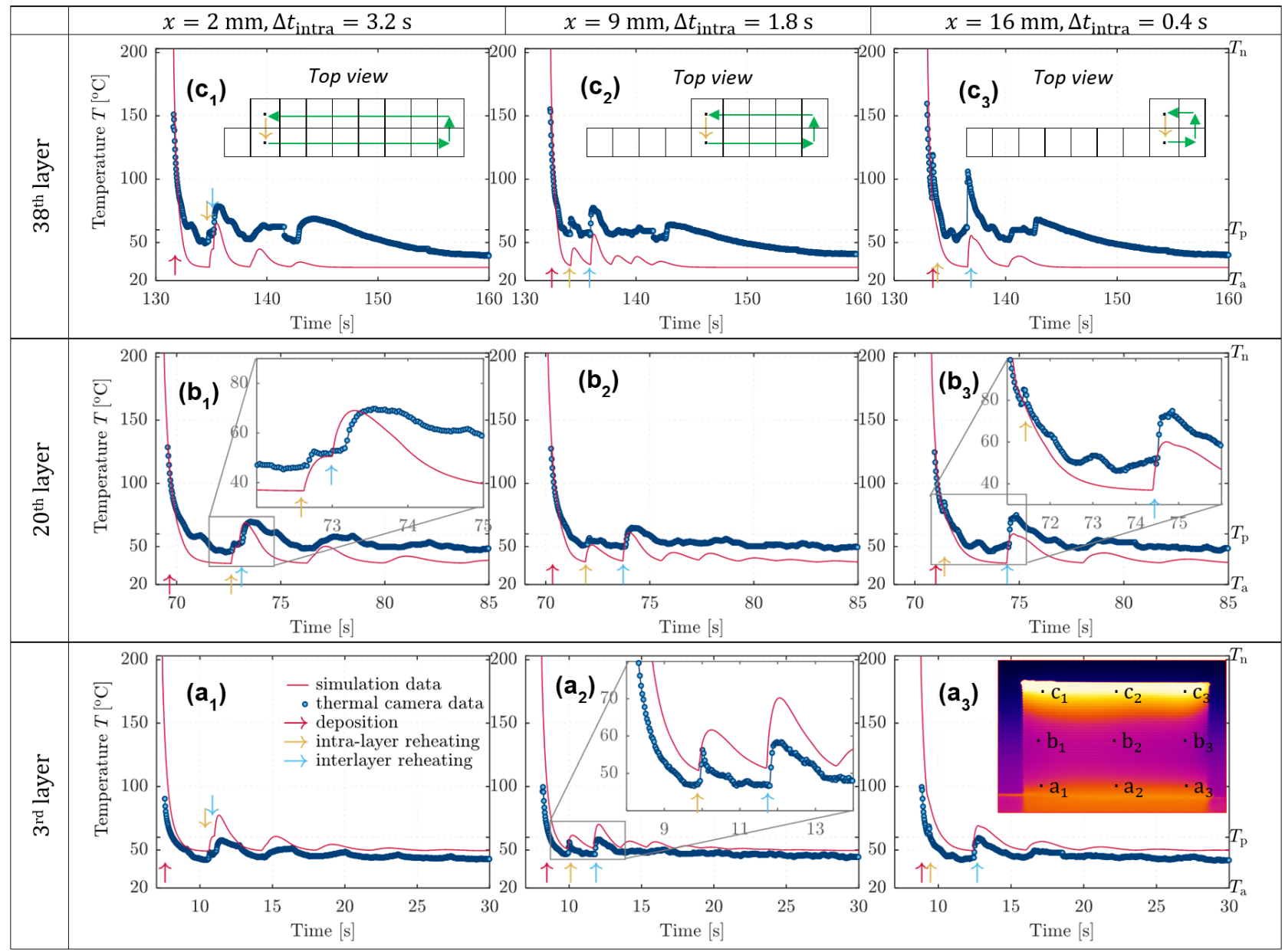

Fig. 2. Experimental and numerical temperature profiles at 9 characteristic locations. As from Fig.1, these locations are labelled, $\mathrm{a}_{1}, \mathrm{~b}_{2}$ and $\mathrm{c}_{3}$, in which, $\mathrm{a}, \mathrm{b}$ and $\mathrm{c}$ indicate the $3^{\mathrm{rd}}, 20^{\text {th }}$ and $38^{\text {th }}$ layers, while 1,2 and 3 indicate $x$ coordinates of 2 , 9 , and $16 \mathrm{~mm}$, respectively. 


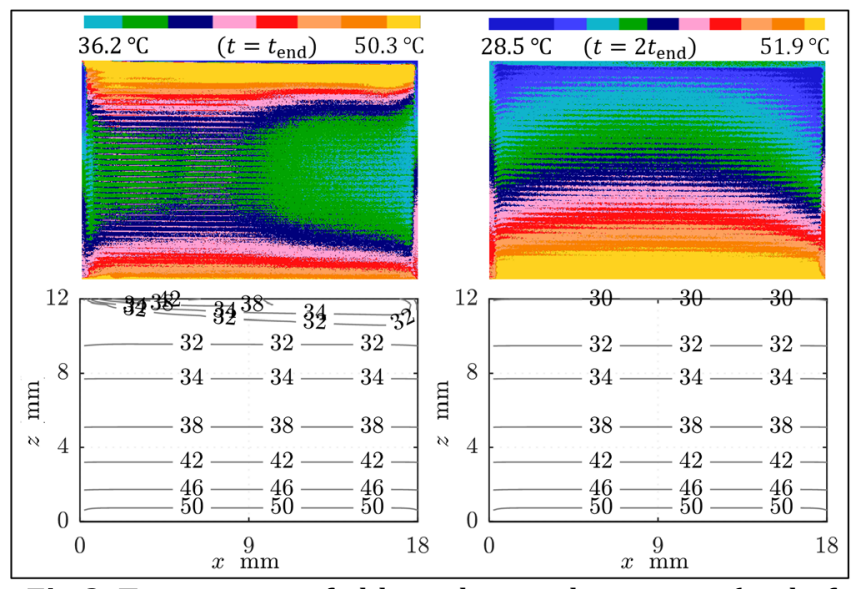

Fig.3. Temperature fields in the $x-z$ plane at $t_{\text {end }}$ (end of printing) and $2 t_{\text {end. }}$

The experimental and numerical temperature fields were finally compared with each other in the time domain.

Fig. 3 shows thermograms and numerical predictions of the temperature distributions in the $x-z$ plane at time $t_{\text {end }}$ (i.e. once fabrication is completed) and at time $2 t_{\text {end }}$ (i.e. after a period of time twice the fabrication time, 280s); it is expected that at time $2 t$ end the system has reached a steady state.

The numerical predictions reach the thermal equilibrium very fast; overall, the values are underestimated as compared to the experimental data. On the contrary, the experimental thermograms show interesting profiles: i) a quasi-symmetric temperature distribution with respect to the central axis and the central line at time $t_{\text {end; }}$ and ii) a U-

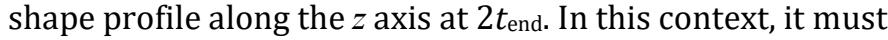
be noted, that those distributions are the result of the particular setting with respect to the temperature of the build platform and the environment.

\section{Discussion}

Sect. 3 shows that, although the computed and measured temperature patterns agree with each other to a high degree, discrepancies exist between the absolute values. This can be due to a number of factors discussed below. Their relative contribution might be variable, depending on the production time $t$, and a critical estimation of those aspects will be subject of future work.

Abrupt temperature changes and sampling frequency. Cooling and heating of a filament segment can be very fast in FFF due to the small characteristic dimensions [3] (layer thickness, and track diameter are typically in the order of 100-300 $\mu \mathrm{m}$ ), and the particular conditions (fan ON, open build chamber, build platform at low temperature, etc.). As an example, a local cooling rate up to $640^{\circ} \mathrm{C} / \mathrm{s}$ and up to 350 ${ }^{\circ} \mathrm{C} / \mathrm{s}$ are computed using the numerical model and measured by the thermal camera for the given test, respectively. Hence, taking into account the camera sampling rate of $32 \mathrm{~Hz}$, i.e. a data acquisition every $0.031 \mathrm{~s}$, a measurement error between $10-20{ }^{\circ} \mathrm{C}$ can be estimated. This partially explains the differences in absolute value, especially at deposition of the filament segment, whereupon discrepancies between $50-100{ }^{\circ} \mathrm{C}$ were registered depending on the particular location (bottom or top layers, see also Fig.2 patterns $\mathrm{a}_{1}-\mathrm{a}_{2}-\mathrm{a}_{3}$ and $\left.\mathrm{c}_{1}-\mathrm{C}_{2}-\mathrm{c}_{3}\right)$.

Size change of the source of radiation. Due to the additive nature of the FFF process, the object enlarges during fabrication, and it covers a larger portion of the FOV of the camera with the progressive building. Hence, the in-process intensity of the relevant signal rises, and the data measured by the camera at the beginning of production might be underestimated. This would explain the general trend noticed in Fig. 2, by which the measured temperature profiles increase overall with the number of the deposited layers (Fig. 2, see for instance the overall trend of temperature at location $\mathrm{a}_{1}, \mathrm{~b}_{1}$ and $\mathrm{c}_{1}$ and compare them). As an example, the temperature differences at the time of deposition decrease from about $100{ }^{\circ} \mathrm{C}$ to about $50{ }^{\circ} \mathrm{C}$ with the number of deposited layers (see Fig.2, patterns a1-a2-a3 and $\mathrm{c}_{1}-\mathrm{c}_{2}-\mathrm{c}_{3}$ at the deposition point (red arrow)).

Emissivity calibration and angle of view. The material emissivity $\varepsilon$ increases with the temperature of the irradiating object and decreases with the angle of incidence of the signal on the camera detector (view angle, $\alpha$ ) (Haitjema, [13]). In this research, $\varepsilon$ was calibrated for a PLA track deposited onto the build platform heated at $60^{\circ} \mathrm{C}$, and it was fixed at 0.78 . Hence, the measurement of high temperature data could be underestimated; contrariwise, the acquisition of the low temperature data could be overestimated. To compensate for this aspect, correction to an empirical temperature-dependent emissivity function would be necessary. Similar considerations can be made regarding the emissivity-view angle dependency.

Model boundary conditions and energy loss. In general, the numerically computed cooling and heating rates are overestimated in comparison to the experimental data. This is reasonable giving the first type boundary conditions, being the energy loss due to convention not optimized and radiation neglected. Also, the model assumes the part as a $100 \%$ full block; instead, entrapped air due to inter-/intralayer porosity play a role in the overall thermal energy balance. An accurate estimation of the energy loss due to thermal radiation (estimated by the authors to be comparable to the energy loss due to convection) is of primary importance when dealing with low cooling rate strategy, e.g. in the presence of hot environment or temperature controlled closed chambers, in order to promote filament bonding (e.g. in high-tech polymers as PEEK) and/or to minimize object warping and delamination [7].

\section{Application example}

The fabricated samples were cut along the relevant $y-z$ planes $\left(x \sim 2,9,16 \mathrm{~mm}\right.$, and $\Delta t_{\text {intra }} \sim 3.2, \sim 1.8, \sim 0.4 \mathrm{~s}$ respectively); the cross sections were polished and then inspected under a microscope for quality assurance (Fig.4). The lengths of the intra- and inter-layer bond lines were measured and statistically compared. Table 2 lists the results obtained. It shows that the plane characterised by the shortest intra-layer time presents the longest bond 
lines; the differences are statistically significant. Looking at the temperature profiles (Fig.2) of the locations $a_{3}, b_{3}$, and $c_{3}$, re-heating takes place quickly after deposition (i.e. fast occurrence of consecutive peaks from time of deposition) and when the material is still above $60{ }^{\circ} \mathrm{C}$ ( $T_{\mathrm{g}}$ of PLA). In addition, those elements of material remain hotter than 60 ${ }^{\circ} \mathrm{C}$ for relatively longer period ( $>1 \mathrm{~s}$ ) as compared to others. The findings are in line with the literature, by which coalescence takes place above $T_{\mathrm{g}}$ and it is promoted at higher temperatures $[2,7]$.

\section{Table 2}

Inter-/intra-layer bond lines (or neck size) for various $\Delta t_{\text {intra. }}$

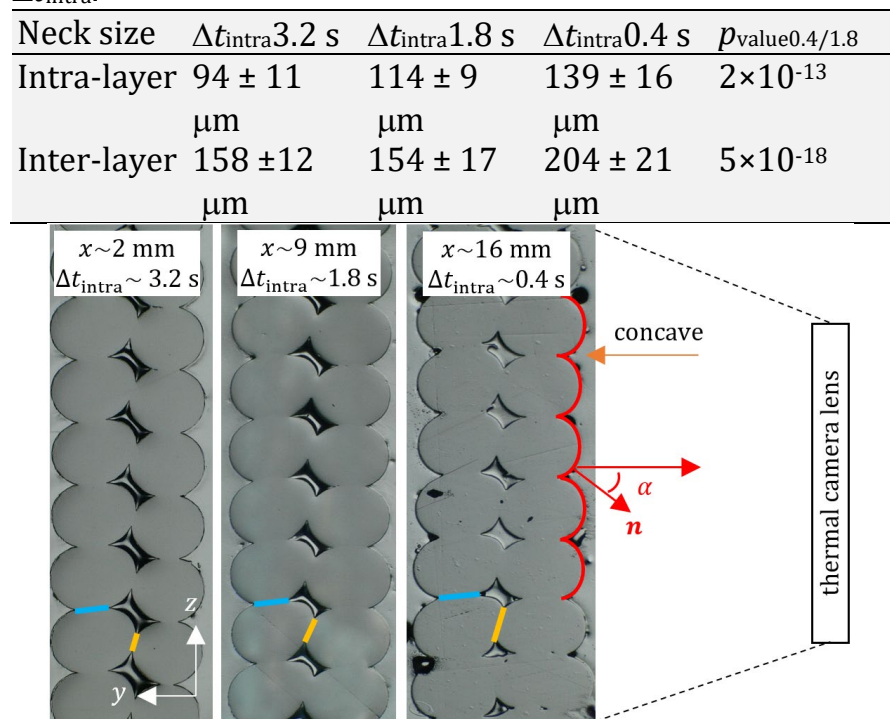

Fig. 4. Microscopic left view of sample cross-sections along the central $y-z$ plane $\left(x \sim 9 \mathrm{~mm}, \Delta t_{\text {intra }} \sim 1.8 \mathrm{~s}\right)$ and along two $y-z$ planes located at edges of the sample $(x \sim 2 \mathrm{~mm}$ and 16 $\mathrm{mm}$, characterised by a $\Delta t_{\text {intra }} \sim 3.2 \mathrm{~s}$ and $0.4 \mathrm{~s}$ ). The bond length at the inter-layer interface is highlighted in blue, while the inter-layer neck size is marked in yellow. The concept of camera view angle and concave regions is also schematically represented.

\section{Conclusions}

This work presents an infrared based set-up capable of capturing spatial and temporal variations of temperature during the progressive printing of a FFF part. This enables the development of a combined experimental and numerical approach for in-process monitoring of fused filament fabrication methods. Accurate acquisition via infrared thermography reveals to be challenging, especially with respect to i) calibration of the emissivity, which is a function of temperature and view angle, and ii) variation of the signal intensity due to the additive nature of the process. The current numerical model should also be improved to better account for the contribution of radiation and convention in FFF. A correlation between the acquired temperature profiles and bond lengths could be found, as a first proof that temperature information can be used for in-process inspection. Additional experiments are necessary for a fully validation of the method, e.g. in case of parts larger than the FOV and with complex geometry.

\section{Acknowledgements}

Part of the work has been performed in the framework of the project: FLAMINCO-Polymer filaments for additive manufacturing of industrial componentsAIO/HBC.2017.0325. The authors are also thankful to Loren De Vogelaer for his technical support.

\section{References}

[1] Galantucci L.M., Lavecchia F., Percoco G., 2018 Study of compression properties of topologically optimized FDM made structured parts, CIRP Annals 57: 243-246

[2] Bellehumeur C., Li L., Sun Q., Gu P., 2004, Modeling of Bond Formation Between Polymer Filaments in the FDM Process, J. Manuf. Proc., 6/2;170-178

[3] S. Volz (Ed.), 2007, Micro and Nanoscale Heat Transfer. Springer, 2007

[4] Faes M., Ferraris, E. Moens D., 2016, Influence of interlayer cooling time on the quasi-static properties of $\mathrm{ABS}$ components produced via FDM, Proc. CIRP, 42:748-753

[5] Sun Q., Rizvi G. M., Bellehumeur C. T., P. Gu, 2008, Effect of processing conditions on the bonding quality of FDM polymer filaments, Rapid Prototyp. J., 14/2: 72-80

[6] Costa S.F., Duarte F.M., Covas J.A., 2017, Estimation of filament temperature and adhesion development infused deposition techniques, J. Mat. Proc Tech 245: 167-179

[7] Rodriguez J.F., Thomas J.P., Renaud J.E., 2003. Design of fused-deposition ABS components for stiffness and strength. J. Mech. Des. 125: 545-551

[8] Bellini A., Güceri S., 2003, Mechanical characterization of parts fabricated using fused deposition modelling. Rapid Prototyp. J. 9: 252-264

[9] Kousiatza C., Karalekas D., 2016, In-situ monitoring of strain and temperature distributions during fused deposition modelling process, Mater. Des. 97: 400-406

[10] Wolszczak P., Lygas K., Paszko M., Wach R. A., 2018, Heat distribution in material during fused deposition modelling, Rapid Prototyp. J., 24/3: 615-622

[11] Malekipour E., Attoye S., Mounayri H., 2018, Investigation of Layer Based Thermal Behavior in FDM by Infrared Thermography, Proc. Manuf., 26:1014-1022

[12] Zhang J., Wang X.Z., Yu W.W., Deng Y.H., 2017, Investigation of the influence of process conditions on the temperature variation in FDM, Mater. Des., 130: 59-68

[13] Haitjema H., 1989, Spectrally selective tinoxide and indiumoxide coatings, PhD Thesis, Delft. Fig. 2.24-2.27 